University of Nebraska - Lincoln

DigitalCommons@University of Nebraska - Lincoln

2015

\title{
White Matter Differences Between Multiple System Atrophy (Parkinsonian Type) and Parkinson's Disease: A Diffusion Tensor Image Study
}

L. Ji

Yingying Wang

Nanjing Medical University

J. Shi

Follow this and additional works at: https://digitalcommons.unl.edu/specedfacpub

Part of the Neuroscience and Neurobiology Commons, and the Special Education and Teaching Commons

This Article is brought to you for free and open access by the Department of Special Education and Communication Disorders at DigitalCommons@University of Nebraska - Lincoln. It has been accepted for inclusion in Special Education and Communication Disorders Faculty Publications by an authorized administrator of DigitalCommons@University of Nebraska - Lincoln. 


\title{
White Matter Differences Between Multiple System Atrophy (Parkinsonian Type) and Parkinson's Disease: A Diffusion Tensor Image Study
}

\author{
L. Ji, ${ }^{1}$ Y. Wang, ${ }^{2,5}$ D. Zhu, ${ }^{1}$ W. Liu, ${ }^{3}$ and J. Shi ${ }^{1,4}$
}

1 Department of Neurology, Nanjing Brain Hospital, Nanjing Medical University, No. 264 Guangzhou Road, Nanjing 210029, PR China

2 Division of Developmental Medicine, Department of Medicine, Boston Children's Hospital, 300 Longwood Avenue, Boston, MA 02115, United States

3 Department of Radiology, Nanjing Brain Hospital, Nanjing Medical University, No. 264 Guangzhou Road, Nanjing 210029, PR China

4 Department of Neurology, School of Medicine, Nanjing University, No. 22 Hankou Road, Nanjing 210093, PR China

5 Current affiliation - University of Nebraska-Lincoln, Lincoln, NE, USA; yingying.wang@unl.edu

Corresponding author - J. Shi, Department of Neurology, Nanjing Brain Hospital Affiliated to Nanjing Medical University, 264 Guangzhou Road, Nanjing 210029, PR China. Tel: +86-025-82296370; fax: +86-025-83719457. E-mail address: profshijp@163.com (J. Shi).

\begin{abstract}
Published in Neuroscience 305 (2015), pp 109-116.

doi 10.1016/j.neuroscience.2015.07.060

Copyright (c) 2015 IBRO. Published by Elsevier Ltd. Used by permission.

Accepted 21 July 2015; published 26 July 2015.
\end{abstract}

The clinical differential diagnosis between the Parkinson variant of multiple system atrophy (MSA-P) and Parkinson's disease (PD) is difficult in early stages. To identify objective markers for differential diagnosis, we combined the novel tract-based spatial statistics (TBSS) and region of interest (ROI) analyses for the first time to 
investigate three groups (15 MSA-P, 20 PD patients and 20 controls) with diffusion tensor imaging data. By TBSS, we performed pairwise comparisons of fractional anisotropy (FA), mean diffusivity, radial diffusivity (RD) and axial diffusivity maps. The clusters with significant differences between MSA-P and PD were used as ROIs for further analyses. FA/RD values in bilateral corticospinal tract (CST) and left anterior thalamic radiation (ATR) in MSA-P were significantly different from PD or controls, and significantly correlated with clinical data. These findings indicated that the abnormalities of left ATR and bilateral CST were specific for MSA-P relative to PD or controls, and seemed to be promising for differential diagnosis. Furthermore, it may be useful for severity assessment of MSA-P.

Keywords: diffusion tensor imaging, multiple system atrophy, Parkinson's disease, region of interest, tract-based spatial statistic

\author{
Abbreviations: \\ ADC apparent diffusion coefficients \\ ATR anterior thalamic radiation \\ CST corticospinal tract \\ DTI diffusion tensor image \\ DWI diffusion weighted image \\ FA fractional anisotropy \\ IFOF inferior fronto-occipital fasciculus \\ MCP middle cerebellar peduncle \\ MD mean diffusivity \\ MSA multiple system atrophy \\ PD Parkinson's disease \\ $\mathrm{ROI}$ region of interest \\ SLF superior longitudinal fasciculus \\ TBSS tract-based spatial statistic analysis \\ UPDRS Unified Parkinson's Disease Rating Scale
}

\title{
Introduction
}

Multiple system atrophy (MSA) is a neurodegenerative disease that can be divided into two clinical subtypes according to whether MSA has predominant cerebellar symptoms (MSA-C) or predominant parkinsonian symptoms (MSA-P) (Gilman et al., 2008). MSA-P has similar symptoms and signs to Parkinson's disease (PD), especially in the early stages. Early differentiation between MSA-P and PD has important prognostic and therapeutic implications. During the past decade, several tools have been developed to address this issue. In conventional magnetic resonance imaging $(\mathrm{MRI})$, the typical radiographic changes such as "slit-like" marginal hyperintensity of the putamen and "hotcross bun" sign in pontine images, usually appear at the advanced 
stage of this disease. Thus, many researchers have recently focused on other non-invasive technologies, such as diffusion weighted image (DWI) and diffusion tensor image (DTI).

Studies comparing DWI changes found higher apparent diffusion coefficients (ADC) or lower fractional anisotropy (FA) values in regions associated with clinical symptoms, such as pons, cerebellum, middle cerebellar peduncle (MCP) and putamen in MSA-P than in PD or controls (Schocke et al., 2004; Nicoletti et al., 2006; Ito et al., 2007; Kollensperger et al., 2007). In theory, DTI can provide more precise details on tissue microstructure than DWI (Mori and Barker, 1999). DTI exploits the random diffusion motion of water molecules in vivo (Moseley, 2002), and reveals important information about the status of neuronal fiber tracts that is not evident on conventional MRI. Several measures can be extracted from DTI analyses, including FA, mean diffusivity (MD), axial diffusivity (AD) and radial diffusivity (RD). FA is considered a valid measure of white matter structural integrity, sensitive to anomalies in axonal density, diameter, myelination and coherence of directional alignment of fibers within white matter tracts (Pierpaoli and Basser, 1996; Le Bihan et al., 2001), while MD represents a directionally independent measure of the average diffusivity that reflects the degree of myelination, interstitial space and axonal density (Norris, 2001). AD measures diffusion parallel to the white matter tracts. RD appears to reflect diffusion perpendicular to white matter tracts. It is recommended to use multiple diffusion tensor measures to better characterize the tissue microstructure (Alexander et al., 2007).

Previous DTI studies have used conventional MRI to set region of interest (ROI) in intracranial structures that are vulnerable in MSA or PD and related to the clinical symptoms, then obtained quantitative data for further analyses. They have demonstrated significant reduction of FA values in MCP, pontine and cerebellar white matter, and putamen in MSA-P compared with PD (Ito et al., 2007; Nilsson et al., 2007; Nair et al., 2013).

The novel tract-based spatial statistics (TBSS) method which combines the strength of both voxel-based and tractography-based analyses has received more attention recently (Smith et al., 2006). It does not require smoothing and allows for higher spatial comparability. Furthermore, it is available for the alignment and registration of major fiber bundles between different subjects, so as to achieve more accurate group comparisons. This approach is appropriate for 
DTI group analyses focusing on deep white matter fiber tracts (Smith et al., 2004; Woolrich et al., 2009). In recent studies, TBSS has been shown to improve sensitivity for detecting white matter diffusion changes, even with relatively small sample size $(n<30)$ (Focke et al., 2008; Yeh et al., 2009).

However, TBSS has rarely been performed in patients with MSA. One study reported significantly different $A D C$ values in the vicinity of the putamen between PD and MSA-P patients via TBSS algorithm (Cnyrim et al., 1997). Hence, it is hypothesized that the combined use of TBSS and ROI is more accurate for characterizing differences between MSA-P and PD.

After detecting specific micro-structural white matter alterations using the TBSS method, we performed a more detailed investigation of the alteration using the ROI method. Correlation between diffusion indices and clinical data was tested by ROI methods.

\section{Experimental Procedures}

\section{Subjects}

DTI scans were initially acquired from 18 patients with MSA-P. However, two patients were excluded due to large motion artifacts, and one patient was discarded because of the presence of infarction. Therefore, a total of 15 MSA-P patients (13 out of 15 patients fulfilled the criteria for 'probable MSA' and two were classified as 'possible MSA') (Gilman et al., 2008), 20 age- and sex-matched patients with PD (Hughes et al., 1992) and 20 age- and sex-matched healthy volunteers were included in the final analyses (see Table 1). All these subjects were selected randomly by our experienced movement disorder specialist. Patients with MSA-P and PD were assessed using the motor part of Unified Parkinson's Disease Rating Scale (UPDRS), Hoehn-Yahr $(\mathrm{H}-\mathrm{Y})$ scale. Patients with MSA-P were also assessed by Unified Multiple System Atrophy Rating Scale (UMSARS), as it was conducive to classification. The study was approved by our regional research ethics committee. Written informed consent was obtained from all participants. All cases were recruited from Nanjing Brain Hospital, from January 2011 to August 2013. 


\section{MRI protocol}

DTI was performed on a 3-Tesla Siemens Verio scanner with an 8-channel radio frequency coil, using a single-shot spin-echo diffusion-weighted echo-planar pulse sequence at 3-mm section thickness with no gap (TR/TE $=8800 / 88 \mathrm{~ms}$; acquisition matrix $=128 \times 128)$. Thirty diffusion-weighted volumes with gradient encoding applied in 30 non collinear directions and $b=1000 \mathrm{~s} / \mathrm{mm}^{2}$ were used to image the entire brain with a $23-\mathrm{cm}$ square field of view (resolution $=1.8 \mathrm{~mm}$ $\times 1.8 \mathrm{~mm} \times 3 \mathrm{~mm}$ ). T1 and T2 weighted images were also obtained with no diffusion gradient $\left(b=0 \mathrm{~s} / \mathrm{mm}^{2}\right)$, as the latter is conducive to further exclusion of other organic diseases such as tumors, infarction and hydrocephalus.

\section{Image analysis}

Images were processed and analyzed using the FSL (FMRIB Software Library, FMRIB, Oxford, UK) software package (Smith et al., 2004; Woolrich et al., 2009; Jenkinson et al., 2012). First, the FDT (FMRIB's Diffusion Toolbox) "eddy_correct" function was used to reduce distortion and the effect of head movement. After that, a brain mask was created from the first $b^{0}$ image using the BET (Brain Extraction Tool) (the fractional intensity threshold is 0.2). Finally, we computed the diffusion maps using the FDT tool "dtifit" to fit the tensor model at each voxel.

Voxelwise analysis was conducted using the FSL tool "TBSS". Data were projected on a common pseudoanatomical skeleton, therefore smoothing could be avoided, and we could carry out the voxelwise statistics without matching every voxel in different subjects. All subjects' FA data were first aligned to the FMRIB58_FA standard-space image using the nonlinear registration tool. Next, the TBSS script created the mean FA image, and skeletonized it using a protocol that searches and labels the skeleton voxels with maximum FA intensity along the perpendicular direction (breadth) of a white matter tract. To make sure that gray matter, ventricle and cerebrospinal fluid were not included, a threshold of 0.2 was set. Finally, the registered FA images were projected onto the skeleton before running the voxelwise cross-subject stats. The MD, RD and AD images were performed in the same way as described above without the initial registrations. 


\section{Statistical analysis}

Statistical analyses were performed with SPSS for Windows, 13.0 (SPSS Inc., Chicago, IL, USA). The significance level was set at $P<0.05$. We first demonstrated the normality distribution of the clinical parameters by the Kolmogorov-Smirnov test. The age was normally distributed. Then, a one-way analysis of variance (ANOVA) followed by post hoc Bonferroni correction was performed for comparison of age at examination. Differences in sex distribution among MSA-P, PD and controls were evaluated with a chi-square test. To assess the differences in disease duration, $\mathrm{H}-\mathrm{Y}$ scale and UPDRS motor scores between patient groups, the Mann-Whitney $U$-test was employed.

To determine the diffusion skeleton voxels that were significantly different between the MSA-P and PD groups, between the PD and control groups, and between the MSA-P and control groups, we estimated two contrasts by the FSL tool "General Linear Model (GLM)". The purpose of the two contrasts is to test whether the means of two groups differ. Significance of contrast 1 indicates that mean (group 1)>mean (group 2), or the first group's mean is larger than the second. Similarly, significance of contrast 2 indicates mean (group 1)<mean (group 2). Age was entered into the analysis as a covariate to ensure that any observed differences of diffusion indices between groups were independent of age. Next, a non-parametric group model using "randomize" in FSL (Nichols and Holmes, 2002) was performed with 5000 permutations. The option threshold-free cluster enhancement (TFCE) (Smith and Nichols, 2009) — not having to define a cluster-forming threshold or perform smoothing - was applied for multiple comparisons correction. The results were viewed and overlaid onto the standard brain using Fslview at a family wise error (FWE) corrected threshold of $P<0.05$. The voxels in the significant clusters were localized by the "JHU ICBM-DTI-81 White Matter Labels Atlas" and "JHU White-Matter Tractography Atlas" (Mori et al., 2008). The clusters were also shown in Montreal Neurological Institute (MNI) coordinates (mm).

For further quantitative analysis, circular ROIs, $4 \mathrm{~mm}$ in diameter for each, were selected at clusters showing significant FA or RD difference between MSA-P and PD. The regional mean values of the diffusion indices were calculated from normalized images. The ROIs were also applied in normalized images of controls, which were used as a 
reference. We then used non-parametric (Kruskal-Wallis) analysis of variance to test for differences among three groups, followed by the Mann-Whitney $U$ test for pairwise comparison. A Bonferroni correction was applied for the number of comparisons ( $n=3$ : [MSA-P vs PD, MSA-P vs controls, $P D$ vs controls], setting the level of significance at $P<0.05 / 3=0.016$ ).

In the MSA-P and PD patient groups separately and combined, Spearman's rank-order correlation analyses were used to test the correlations between regional FA/RD changes and clinical data by ROI analysis.

\section{Results}

\section{Group characteristics}

Table 1 shows demographic and clinical data of MSA-P patients, PD patients and controls. There were no significant differences in age and sex among the three groups. $\mathrm{H}-\mathrm{Y}$ scale and UPDRS motor score did not show any differences between patient groups. However, a small but statistically significant difference in disease duration between the patient groups was found.

Table 1. Demographic and behavioral characteristics of the included participants

\begin{tabular}{lrrrr} 
Characteristics & $\begin{array}{r}\text { MSA-P } \\
(n=15)\end{array}$ & $\begin{array}{r}P D \\
(n=20)\end{array}$ & $\begin{array}{r}\text { Control } \\
(n=20)\end{array}$ & $P^{*}$ \\
\hline Age at DTI, mean (SD) & $59.87(7.20)$ & $64.20(7.37)$ & $59.95(4.88)$ & 0.124 \\
Gender (male: female) & $9: 6$ & $11: 9$ & $10: 10$ & 0.840 \\
Disease duration at DTI, median (range) & $2(1-5)$ & $5(1-8)$ & NA & 0.048 \\
H-Y, median (range) & $2(1-3)$ & $2(1-4)$ & NA & 0.090 \\
UPDRS motor score, median (range) & $28(10-40)$ & $32(11-44)$ & NA & 0.356 \\
$\quad$ (off medication) & & & & \\
\hline
\end{tabular}

HY: Hoehn and Yahr scale; UPDRS: Unified Parkinson Disease Rating Scale; NA: not applicable; SD: standard deviation. 


\section{White matter difference assessed by using TBSS and ROI}

Significantly decreased FA values in MSA-P compared with PD groups were noted in the bilateral corticospinal tract (CST) and left anterior thalamic radiation (ATR). No increased FA values were found. Higher $R D$ values were found in the bilateral CST and left ATR in MSA-P patients compared with PD. In addition, some clusters representing ATR and CST appeared in the region of MCP (Table 2 and Fig. 1).

Based on the TBSS results, regional mean values of diffusion indices from these clusters in Table 2 were calculated. FA values in the bilateral CST and left ATR were significantly lower in patients with MSA$P$ than PD $(P<0.001 ; P=0.008)$ or normal controls $(P<0.001 ; P=0.001)$ (Table 3). The bilateral CST and left ATR also showed higher MD values in patients with MSA-P compared with PD $(P<0.001 ; P<0.001)$ or normal controls $(P<0.001 ; P<0.001)$ (Table 3$)$.

Table 2. Clusters showing significant difference of diffusion indices in pairwise comparison

\begin{tabular}{|c|c|c|c|c|c|c|}
\hline \multirow[t]{2}{*}{ White matter location } & \multicolumn{4}{|c|}{ MNI coordinates (mm) } & \multirow[b]{2}{*}{ Difference } & \multirow[b]{2}{*}{$P^{*}$} \\
\hline & Voxels & $x$ & $y$ & $z$ & & \\
\hline \multicolumn{7}{|l|}{$M S A-P$ vs $P D$} \\
\hline \multicolumn{7}{|l|}{ FA } \\
\hline Corticospinal tract $\mathrm{L}$ & 224 & -10 & -32 & -29 & $\mathrm{P}>\mathrm{M}$ & 0.014 \\
\hline Corticospinal tract $\mathrm{R}$ & 139 & 12 & -24 & -33 & $\mathrm{P}>\mathrm{M}$ & 0.018 \\
\hline Anterior thalamic radiation $\mathrm{L}(\mathrm{MCP})$ & 29 & -9 & -45 & -32 & $\mathrm{P}>\mathrm{M}$ & 0.020 \\
\hline \multicolumn{7}{|l|}{ RD } \\
\hline Corticospinal tract $\mathrm{L}$ & 180 & -13 & -32 & -37 & $M>P$ & 0.044 \\
\hline Corticospinal tract $\mathrm{R}$ & 165 & 18 & -52 & -30 & $M>P$ & 0.048 \\
\hline Anterior thalamic radiation $\mathrm{L}(\mathrm{MCP})$ & 346 & -9 & -44 & -33 & $M>P$ & 0.044 \\
\hline \multicolumn{7}{|l|}{ MSA-P vs controls } \\
\hline \multicolumn{7}{|l|}{ FA } \\
\hline Corticospinal tract $\mathrm{L}$ & 315 & -7 & -27 & -34 & $\mathrm{c}>\mathrm{M}$ & 0.008 \\
\hline Superior longitudinal fasciculus $\mathrm{R}$ & 68 & 50 & -1 & 18 & $\mathrm{c}>\mathrm{M}$ & 0.010 \\
\hline Anterior thalamic radiation $\mathrm{R}$ & 65 & 9 & -45 & -32 & $c>M$ & 0.010 \\
\hline \multicolumn{7}{|l|}{ MD } \\
\hline Superior longitudinal fasciculus R (external capsule) & 4531 & 33 & -9 & 7 & $M>c$ & 0.008 \\
\hline Anterior thalamic radiation $\mathrm{L}$ & 394 & -14 & -52 & -27 & $M>c$ & 0.014 \\
\hline Corticospinal tract $\mathrm{L}$ & 141 & -18 & -36 & -36 & $M>C$ & 0.016 \\
\hline \multicolumn{7}{|l|}{$A D$} \\
\hline Superior longitudinal fasciculus $\mathrm{R}$ & 436 & 37 & -32 & 31 & $M>C$ & 0.044 \\
\hline Anterior thalamic radiation $\mathrm{L}$ & 394 & -14 & -52 & -27 & $M>C$ & 0.014 \\
\hline Corticospinal tract $\mathrm{R}$ & 940 & 26 & -10 & 19 & $M>c$ & 0.022 \\
\hline \multicolumn{7}{|l|}{ PD vs controls } \\
\hline \multicolumn{7}{|l|}{ FA } \\
\hline Body of corpus callosum & 3190 & 9 & 16 & 21 & $c>P$ & 0.044 \\
\hline
\end{tabular}

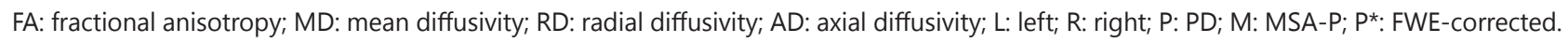




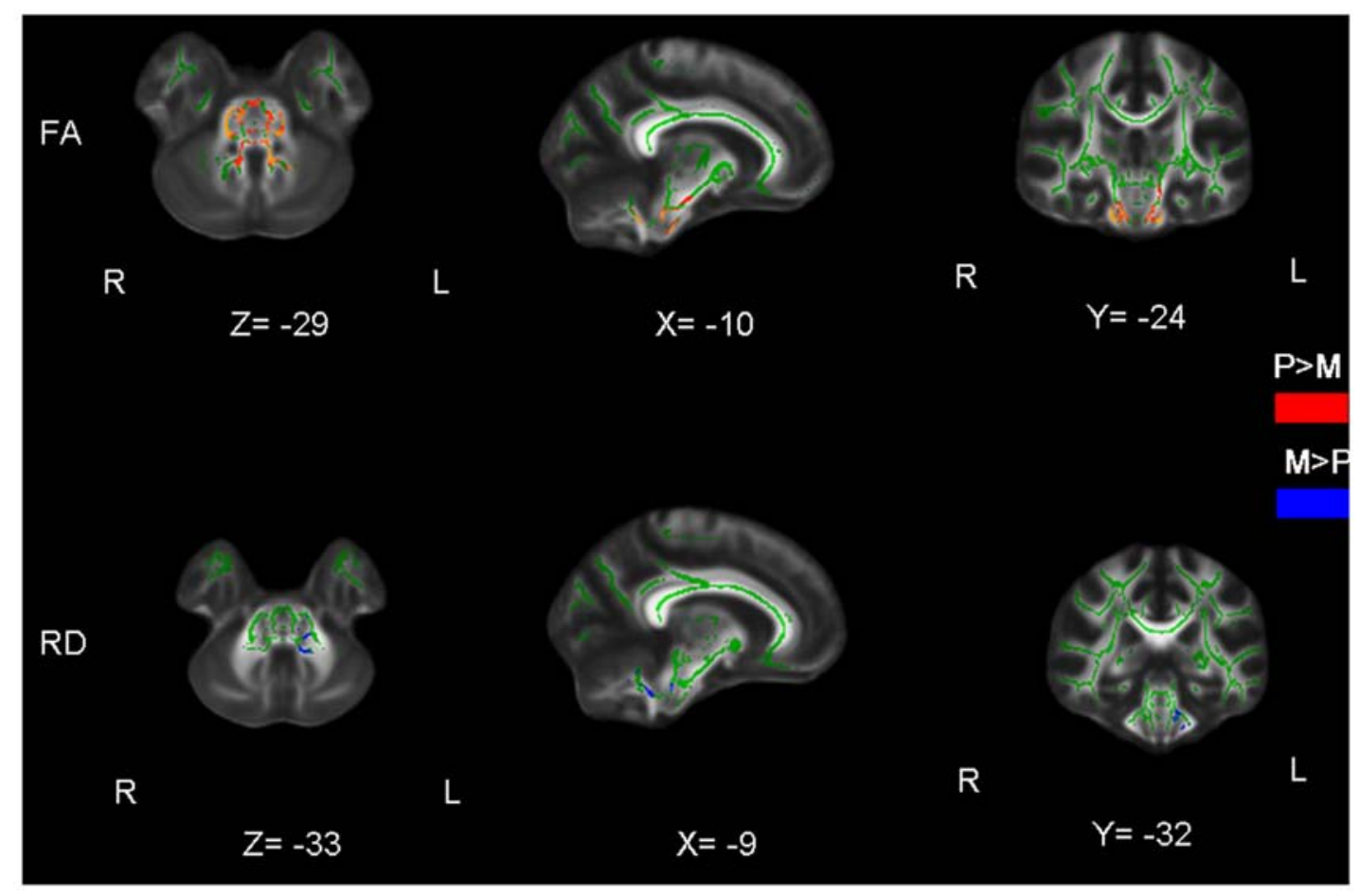

Fig. 1. TBSS results showing significant difference in $F A / R D$ values between MSA-P and PD $(P<0.05)$ corresponding to Table 2. Results are superimposed on fiber skeleton (green) and overlaid on the FMRIB FA $1 \mathrm{~mm}$ template. FA: fractional anisotropy; RD: radial diffusivity; L: left; R: right; P: PD; M: MSA-P.

Table 3. Comparison of regional mean diffusion indices in patients and controls

\begin{tabular}{|c|c|c|c|c|c|c|}
\hline & \multirow[t]{2}{*}{$M S A-P$} & \multirow[t]{2}{*}{$P D$} & \multirow[t]{2}{*}{$C N$} & \multicolumn{3}{|l|}{$P$ value } \\
\hline & & & & $\begin{array}{r}\text { MSA-P } \\
\text { vs PD }\end{array}$ & $\begin{array}{r}\text { MSA-P } \\
\text { VS CN }\end{array}$ & $\begin{array}{r}P D \text { vs } \\
C N\end{array}$ \\
\hline \multicolumn{7}{|l|}{$F A$} \\
\hline Left CST & $0.388(0.200-0.508)$ & $0.517(0.215-0.614)$ & $0.515(0.423-0.628)$ & $<0.001$ & $<0.001$ & NS \\
\hline Right CST & $0.365(0.239-0.530)$ & $0.514(0.186-0.668)$ & $0.484(0.376-0.559)$ & $<0.001$ & $<0.001$ & NS \\
\hline Left ATR & $0.433(0.217-0.564)$ & $0.553(0.487-0.428)$ & $0.553(0.454-0.810)$ & 0.008 & 0.001 & NS \\
\hline \multicolumn{7}{|l|}{$R D$} \\
\hline Left CST & $0.683(0.471-1.224)$ & $0.446(0.372-0.603)$ & $0.447(0.083-0.449)$ & $<0.001$ & $<0.001$ & NS \\
\hline Right CST & $0.610(0.479-0.870)$ & $0.478(0.422-0.545)$ & $0.445(0.395-0.524)$ & $<0.001$ & $<0.001$ & NS \\
\hline Left ATR & $0.921(0.548-1.786)$ & $0.565(0.413-1.127)$ & $0.534(0.310-0.670)$ & $<0.001$ & $<0.001$ & NS \\
\hline
\end{tabular}

FA values, $R D$ values $\left(-10^{-3} \mathrm{~mm}^{2} / \mathrm{s}\right)$ are reported as median value (range). $\mathrm{CN}$ : control subjects; NS: no significance.

Statistical significance $(P<0.016)$. 


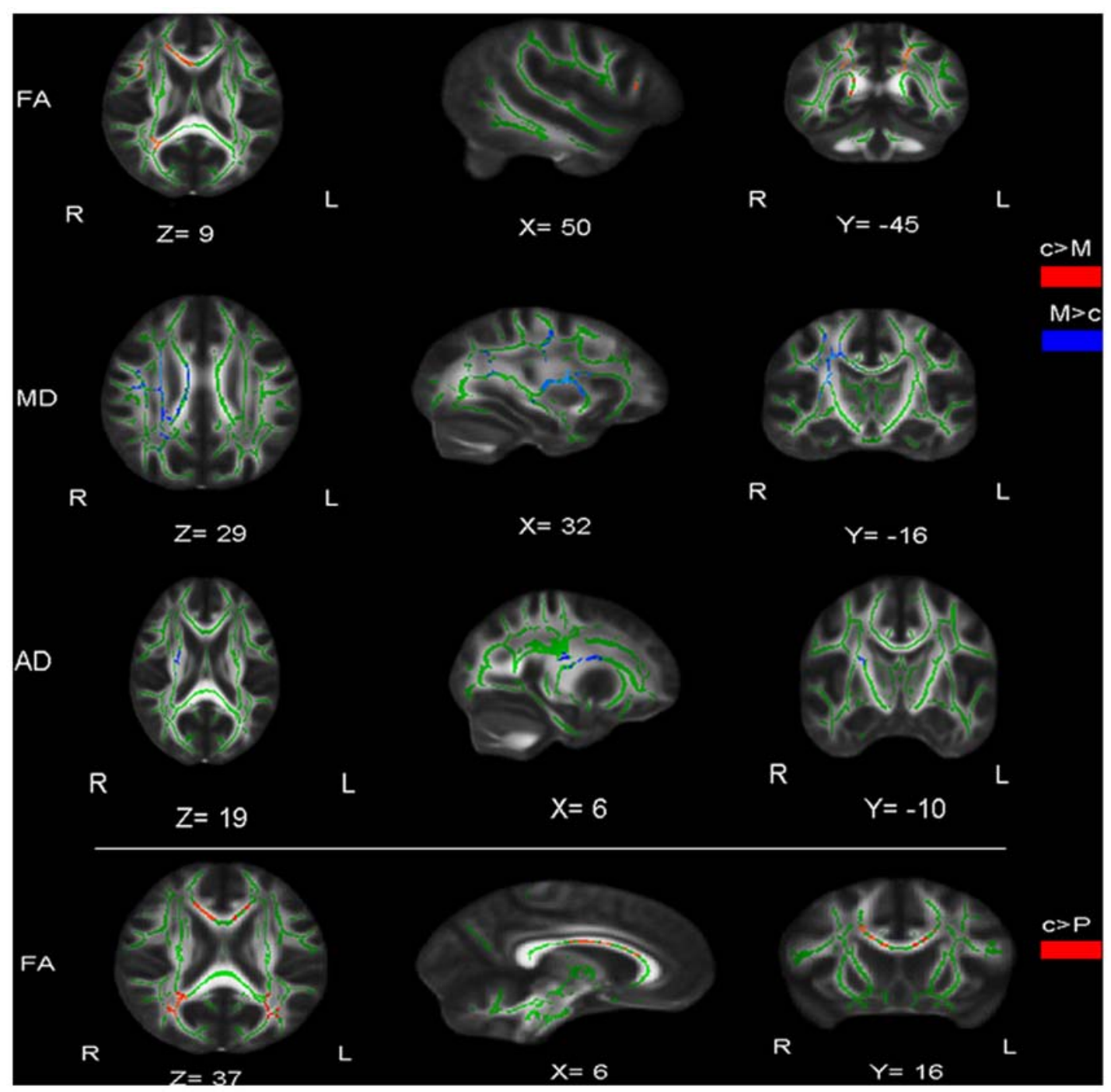

Fig. 2. TBSS results showing significant changes between MSA-P and controls (the upper three rows), between PD and controls (the last row) $(P<0.05)$ corresponding to Table 2. Results are superimposed on fiber skeleton (green) and overlaid on the FMRIB FA $1 \mathrm{~mm}$ template. FA: fractional anisotropy; MD: mean diffusivity; AD: axial diffusivity; L: left; R: right; P: PD; M: MSA-P; c, controls.

Table 2 and Fig. 2 summarize the voxel-wise comparison between MSA-P and controls using TBSS "randomize" method. FA values were significantly lower in the bilateral CST, right ATR and right SLF of patients with MSA-P compared with controls. No increased FA values were found. For MD, the values were increased in the left CST and ATR, right SLF, almost the same white matter tracts as those in which 
FA values were decreased. For $A D$, the values were increased in the left ATR, right SLF and CST (all $P<0.05$ ).

Table 2 and Fig. 2 also show the voxel-wise comparison between PD and controls by TBSS. Significant decreased FA values in the body of corpus callosum were identified in PD patients. No increased FA values were found. For $M D, R D$ or $A D$, no regions with significant differences were found.

\section{Correlations with clinical data by ROI}

Based on the extracted regional mean values from ROls, the correlations were analyzed in the MSA-P and PD patient groups separately and combined. In the separate MSA-P group, there was a significant correlation between disease duration and FA values in the left CST $(r=-0.534, P=0.008)$ as well as left ATR $(r=-0.618, P=0.003)$, between UPDRS motor score and FA values in the right CST $(r=-0.573$, $P=0.016)$ as well as RD values in the left ATR $(r=-0.692, P=0.005)$, and between $\mathrm{H}-\mathrm{Y}$ scale and RD values in the left ATR $(r=0.616, P=0.020)$. In the MSA-P and PD patient groups combined, FA values in the left ATR $(r=-0.520, P=0.004)$ and RD values in the right CST $(r=0.633, P=0.010)$ correlated significantly with $\mathrm{H}-\mathrm{Y}$ scale. FA or RD values did not correlate with any clinical data in the PD group alone.

\section{Discussion}

Our research yielded several major findings. First, comparison of MSA$P$ with PD by TBSS revealed diffusion differences in CST and ATR. A further detailed investigation of these alterations by ROI analysis in the three groups showed that, FA/RD values in bilateral CST and left ATR were significantly reduced in MSA-P than PD or controls. Second, comparison of MSA-P with controls by TBSS showed diffusion abnormalities over a wide area of cerebral white matter. Third, correlation

analysis by $\mathrm{ROI}$ revealed a significant correlation between clinical data and FA/RD values in left ATR as well as bilateral CST.

Previous studies have mostly concentrated on FA or ADC values from DWI to distinguish MSA from PD. They reported higher ADC or lower FA values in basal ganglia or olivopontocerebellar system in patients with MSA-P (Tsukamoto et al., 2012). In our present study, due 
to the application of the whole-brain analysis at the first step instead of the ROI analysis, diffusion abnormalities of some white matter fibers that had never been reported in previous studies were identified. It may aid in understanding the mechanism of these disorders. As noted, our whole-brain analysis by TBSS can exclude the possibility of lesions in other brain structures.

By TBSS, our study demonstrated abnormal FA values in MSA-P. It contradicts previous studies with TBSS reporting no group difference of FA values between MSA-P and PD (Cnyrim et al., 1997). The inconsistencies may be due to differences in DTI parameters, sample size, disease severity, age of patients, duration of disease, patient characteristics, or ethnic differences. According to an epidemiological investigation in Japan, MSA-C was more frequent and MSA-P was less frequent in Japanese populations compared with western populations (Wenning et al., 1997; Watanabe et al., 2002). It was also proposed that the olivopontocerebellar system could be more severely affected in Japanese than in western populations (Watanabe et al., 2004).

In patients with MSA-P compared to PD or controls, changes in diffusion showed a significant decrease of FA and increase of RD in bilateral CST. The deterioration of CST is in agreement with previous reports using transcranial magnetic stimulation or magnetization transfer imaging (Da et al., 2007; Eusebio et al., 2007). Two possible interpretations may explain this phenomenon. First, FA values were decreased at multiple levels of the CST including the MCP. Pallor of myelin, gliosis and glial cytoplasmic inclusions in MCP are one of the primary pathological alterations (Papp et al., 1989; Inoue et al., 1997). Our findings correspond well with pathologically vulnerable areas in MSA. Second, axonal loss in the pyramidal tract due to degeneration of Betz cells in MSA (Tsuchiya et al., 2000) may also result in diffusion abnormality.

Diffusion abnormality of the left ATR in MSA-P patients compared with PD or controls may have several explanations. First, according to the "JHU ICBM-DTI-81 White Matter Labels Atlas", the abnormal tract was located in the region of MCP. Several authors have also reported reduced FA values in this area (Schocke et al., 2002; Shiga et al., 2005). These studies verify the pathological findings that the majority of MSAP patients have moderate or severe degenerative changes in the olivopontocerebellar system (Wenning et al., 1997). Second, this tract connects dorsomedial and anterior thalamic nuclei with the 
prefrontal cortex and it therefore is part of the thalamocortical circuits. Because the thalamocortical circuits are closely associated with the extrapyramidal loop, in our study ATR was correlated significantly with UMSARS motor score. Our findings can be explained from the pathological point of view, that, unlike PD, the major neurodegenerative changes in MSA-P appear outside the substantia nigra. The disrupted thalamocortical connectivity has also been detected in progressive supranuclear palsy (PSP) patients (Whitwell et al., 2011). It can be hypothesized that the same involvement in MSA-P and PSP may cause similar parkinsonian symptoms. In a DWI study of MSA (Pellecchia et al., 2011), a significant increase of Trace (D) was observed at follow-up in the thalamus and frontal white matter, while the clinical severity scores were also significantly increased.

The present analysis also exhibited accented degeneration of the SLF in MSA-P compared with controls. Interestingly, changes of diffusion indices appeared in the region of external capsule adjacent to putamen. Previous studies also described significant degeneration in this area in MSA-P patients (Schocke et al., 2004; Seppi et al., 2006). To our knowledge, SLF is the major cortical association fiber pathway in the brain. It interconnects frontal, temporal and parietal association areas, emphasizing its centrality in many associative or higher brain functions such as spatial working memory, attention, and visuospatial and audiospatial processing (Makris et al., 2005). However, the exact cause of the SLF deficit is still unknown, and further research is needed (Cnyrim et al., 1997). Moreover, a recent MRI study described the degeneration of SLF in PSP and MSA-P patients (Whitwell et al., 2011). These studies suggest that SLF is vulnerable in Parkinson plus syndrome.

The lateralization of DTI differences was obvious in ATR where lower FA and higher RD values appeared only in the left side. It is probably due to the more severe symptoms on the right side in MSA-P patients ( $n=10$ in the MSA-P group). For RD, it is probably because the difference was small and around the threshold value, considering that $P$ values in the left ATR was 0.044 . Another probable reason is that our result exactly reflected laterality of the white matter alteration. Preferably, longitudinal investigations are warranted to elucidate these aspects. As noted, previous studies have reported isolated, unilateral and left-side abnormalities in a few brain areas in MSA (Tir et al., 2009; Mahlknecht et al., 2010). 


\section{Conclusion}

The combined use of TBSS and ROI succeeded in revealing white matter abnormalities in the regional left ATR and bilateral CST, which was specific for MSA-P relative to PD or controls, and might provide a neurobiological basis for MSA pathology. In addition, the diffusion indices of the two tracts changed in accordance with functional disability, which may be helpful in monitoring disease progression in MSA-P patients.

Acknowledgments-This work was supported by the Medical Science and Technology Development Foundation, Nanjing Department of Health (Nos. ZKX08035 and YKK11031) and Nanjing Bureau of Science and Technology (No. 201104009). We acknowledge Chang $\mathrm{Xu}$ and Jie Fan for their assistance in rating scale.

\section{References}

Alexander AL, Lee JE, Lazar M, Field AS (2007) Diffusion tensor imaging of the brain. Neurotherapeutics 4:316-329.

Cnyrim CD, Kupsch A, Ebersbach G, Hoffmann KT (1997) Diffusion tensor imaging in idiopathic Parkinson's disease and multisystem atrophy (Parkinsonian type). Neurodegener Dis 13:1-8.

Da RA, Maia AJ, Da SC, Braga FT, Ferreira NP, Barsottini OG, Ferraz HB (2007) Pyramidal tract degeneration in multiple system atrophy: the relevance of magnetization transfer imaging. Movement Disord 22:238-244.

Eusebio A, Azulay JP, Witjas T, Rico A, Attarian S (2007) Assessment of corticospinal tract impairment in multiple system atrophy using transcranial magnetic stimulation. Clin Neurophysiol 118:815-823.

Focke NK, Yogarajah M, Bonelli SB, Bartlett PA, Symms MR, Duncan JS (2008) Voxel-based diffusion tensor imaging in patients with mesial temporal lobe epilepsy and hippocampal sclerosis. Neurolmage 40:728-737.

Gilman S, Wenning GK, Low PA, Brooks DJ, Mathias CJ, Trojanowski JQ, Wood NW, Colosimo C, Durr A, Fowler CJ, Kaufmann H, Klockgether T, Lees A, Poewe W, Quinn N, Revesz T, Robertson D, Sandroni P, Seppi K, Vidailhet M (2008) Second consensus statement on the diagnosis of multiple system atrophy. Neurology 71:670-676.

Hughes AJ, Daniel SE, Kilford L, Lees AJ (1992) Accuracy of clinical diagnosis of idiopathic Parkinson's disease: a clinico-pathological study of 100 cases. J Neurol Neurosurg Psychiatry 55:181-184. 
Inoue M, Yagishita S, Ryo M, Hasegawa K, Amano N, Matsushita M (1997) The distribution and dynamic density of oligodendroglial cytoplasmic inclusions (GCls) in multiple system atrophy: a correlation between the density of GCls and the degree of involvement of striatonigral and olivopontocerebellar systems. Acta Neuropathol 93:585-591.

Ito M, Watanabe H, Kawai Y, Atsuta N, Tanaka F, Naganawa S, Fukatsu H, Sobue G (2007) Usefulness of combined fractional anisotropy and apparent diffusion coefficient values for detection of involvement in multiple system atrophy. J Neurol Neurosurg Psychiatry 78:722-728.

Jenkinson M, Beckmann CF, Behrens TE, Woolrich MW, Smith SM (2012). Neurolmage 62:782-790.

Kollensperger M, Seppi K, Liener C, Boesch S, Heute D, Mair KJ, Mueller J, Sawires M, Scherfler C, Schocke MF, Donnemilier E, Virgolini I, Wenning GK, Poewe W (2007) Diffusion weighted imaging best discriminates PD from MSA-P: a comparison with tilt table testing and heart MIBG scintigraphy. Mov Disord 22:1771-1776.

Le Bihan D, Mangin JF, Poupon C, Clark CA, Pappata S, Molko N, Chabriat H (2001) Diffusion tensor imaging: concepts and applications. J Magn Reson Imaging 13:534-546.

Mahlknecht P, Hotter A, Hussl A, Esterhammer R, Schocke M, Seppi K (2010) Significance of MRI in diagnosis and differential diagnosis of Parkinson's disease. Neurodegener Dis 7:300-318.

Makris N, Kennedy DN, Mclnerney S, Sorensen AG, Wang R, Caviness VJ, Pandya DN (2005) Segmentation of subcomponents within the superior longitudinal fascicle in humans: a quantitative, in vivo, DT-MRI study. Cereb Cortex 15:854-869.

Mori S, Barker PB (1999) Diffusion magnetic resonance imaging: its principle and applications. Anat Rec 257:102-109.

Mori S, Oishi K, Jiang H, Jiang L, Li X, Akhter K, Hua K, Faria AV, Mahmood A, Woods R, Toga AW, Pike GB, Neto PR, Evans A, Zhang J, Huang H, Miller MI, van Zijl P, Mazziotta J (2008) Stereotaxic white matter atlas based on diffusion tensor imaging in an ICBM template. Neurolmage 40:570-582.

Moseley M (2002) Diffusion tensor imaging and aging - a review. NMR Biomed 15:553-560.

Nair SR, Tan LK, Mohd RN, Lim SY, Rahmat K, Mohd NH (2013) A decision tree for differentiating multiple system atrophy from Parkinson's disease using 3-T MR imaging. Eur Radiol 23:1459-1466.

Nichols TE, Holmes AP (2002) Nonparametric permutation tests for functional neuroimaging: a primer with examples. Hum Brain Mapp 15:1-25.

Nicoletti G, Lodi R, Condino F, Tonon C, Fera F, Malucelli E, Manners D, Zappia M, Morgante L, Barone P, Barbiroli B, Quattrone A (2006) Apparent diffusion coefficient measurements of the middle cerebellar peduncle differentiate the Parkinson variant of MSA from Parkinson's disease and progressive supranuclear palsy. Brain 129:2679-2687. 
Nilsson C, Markenroth BK, Brockstedt S, Latt J, Widner H, Larsson EM (2007) Tracking the neurodegeneration of parkinsonian disorders-a pilot study. Neuroradiology 49:111-119.

Norris DG (2001) The effects of microscopic tissue parameters on the diffusion weighted magnetic resonance imaging experiment. NMR Biomed 14:77-93.

Papp MI, Kahn JE, Lantos PL (1989) Glial cytoplasmic inclusions in the CNS of patients with multiple system atrophy (striatonigral degeneration, olivopontocerebellar atrophy and Shy-Drager syndrome). J Neurol Sci 94:79-100.

Pellecchia MT, Barone P, Vicidomini C, Mollica C, Salvatore E, lanniciello M, Liuzzi R, Longo K, Picillo M, De Michele G, Filla A, Brunetti A, Salvatore M, Pappata $S$ (2011) Progression of striatal and extrastriatal degeneration in multiple system atrophy: a longitudinal diffusion-weighted MR study. Mov Disord 26:1303-1309.

Pierpaoli C, Basser PJ (1996) Toward a quantitative assessment of diffusion anisotropy. Magn Reson Med 36:893-906.

Schocke MF, Seppi K, Esterhammer R, Kremser C, Jaschke W, Poewe W, Wenning GK (2002) Diffusion-weighted MRI differentiates the Parkinson variant of multiple system atrophy from PD. Neurology 58:575-580.

Schocke MF, Seppi K, Esterhammer R, Kremser C, Mair KJ, Czermak BV, Jaschke W, Poewe W, Wenning GK (2004) Trace of diffusion tensor differentiates the Parkinson variant of multiple system atrophy and Parkinson's disease. Neurolmage 21:1443-1451.

Seppi K, Schocke MF, Mair KJ, Esterhammer R, Scherfler C, Geser F, Kremser C, Boesch S, Jaschke W, Poewe W, Wenning GK (2006) Progression of putaminal degeneration in multiple system atrophy: a serial diffusion MR study. Neurolmage 31:240-245.

Shiga K, Yamada K, Yoshikawa K, Mizuno T, Nishimura T, Nakagawa M (2005) Local tissue anisotropy decreases in cerebellopetal fibers and pyramidal tract in multiple system atrophy. J Neurol 252:589-596.

Smith SM, Nichols TE (2009) Threshold-free cluster enhancement: addressing problems of smoothing, threshold dependence and localisation in cluster inference. Neurolmage 44:83-98.

Smith SM, Jenkinson M, Woolrich MW, Beckmann CF, Behrens TE, JohansenBerg H, Bannister PR, De Luca M, Drobnjak I, Flitney DE, Niazy RK, Saunders J, Vickers J, Zhang Y, De Stefano N, Brady JM, Matthews PM (2004) Advances in functional and structural MR image analysis and implementation as FSL. Neurolmage 23(Suppl 1):S208-S219.

Smith SM, Jenkinson M, Johansen-Berg $H$, Rueckert $D$, Nichols TE, Mackay CE, Watkins KE, Ciccarelli O, Cader MZ, Matthews PM, Behrens TE (2006) Tractbased spatial statistics: voxelwise analysis of multi-subject diffusion data. Neurolmage 31: 1487-1505.

Tir M, Delmaire C, le Thuc V, Duhamel A, Destee A, Pruvo JP, Defebvre L (2009) Motor-related circuit dysfunction in MSA-P: Usefulness of combined wholebrain imaging analysis. Mov Disord 24:863-870. 
Tsuchiya K, Ozawa E, Haga C, Watabiki S, Ikeda M, Sano M, Ooe K, Taki K, Ikeda K (2000) Constant involvement of the Betz cells and pyramidal tract in multiple system atrophy: a clinicopathological study of seven autopsy cases. Acta Neuropathol 99:628-636.

Tsukamoto K, Matsusue E, Kanasaki Y, Kakite S, Fujii S, Kaminou T, Ogawa T (2012) Significance of apparent diffusion coefficient measurement for the differential diagnosis of multiple system atrophy, progressive supranuclear palsy, and Parkinson's disease: evaluation by 3.0-T MR imaging. Neuroradiology 54:947-955.

Watanabe H, Saito Y, Terao S, Ando T, Kachi T, Mukai E, Aiba I, Abe Y, Tamakoshi A, Doyu M, Hirayama M, Sobue G (2002) Progression and prognosis in multiple system atrophy: an analysis of 230 Japanese patients. Brain 125:1070-1083.

Watanabe H, Fukatsu H, Katsuno M, Sugiura M, Hamada K, Okada Y, Hirayama M, Ishigaki T, Sobue G (2004) Multiple regional 1H-MR spectroscopy in multiple system atrophy: $\mathrm{NAA} / \mathrm{Cr}$ reduction in pontine base as a valuable diagnostic marker. J Neurol Neurosurg Psychiatry 75:103-109.

Wenning GK, Tison F, Ben SY, Daniel SE, Quinn NP (1997) Multiple system atrophy: a review of 203 pathologically proven cases. Mov Disord 12:133-147.

Whitwell JL, Avula R, Master A, Vemuri P, Senjem ML, Jones DT, Jack CJ, Josephs KA (2011) Disrupted thalamocortical connectivity in PSP: a resting-state fMRI, DTI, and VBM study. Parkinsonism Relat Disorder 17:599-605.

Woolrich MW, Jbabdi S, Patenaude B, Chappell M, Makni S, Behrens T, Beckmann C, Jenkinson M, Smith SM (2009) Bayesian analysis of neuroimaging data in FSL. Neuroimage 45: S173-S186.

Yeh PH, Simpson K, Durazzo TC, Gazdzinski S, Meyerhoff DJ (2009) TractBased Spatial Statistics (TBSS) of diffusion tensor imaging data in alcohol dependence: abnormalities of the motivational neurocircuitry. Psychiatr Res 173:22-30. 